\title{
Aplicaciones de las prácticas ambientales en los comercios ubicados en el asentamiento de Ciudad Porfía (Villavicencio, Colombia)
}

\section{Applications of environmental practices in the shops located in the settlement of Ciudad Porfía (Villavicencio, Colombia)}

CASTRO-GARZÓN, Hernando ${ }^{1}$

PINEDA, Jenifer $S .^{2}$

RODRÍGUEZ, Juan P. ${ }^{3}$

\section{Resumen}

El propósito de este trabajo es determinar los impactos ambientales que generan los comercios ubicados en el asentamiento Ciudad Porfía de la ciudad de Villavicencio, Colombia. El trabajo abordó como principal técnica un método cuantitativo de tipo descriptivo, el cual permitió realizar observación y verificación de antecedentes; en cuanto a la recolección de información se aplicó una herramienta tipo encuesta en 133 establecimientos de comercio cuyos datos obtenidos se procesaron mediante herramientas estadísticas.

Palabras clave: sostenibilidad, impactos, prácticas ambientales, mitigación

\begin{abstract}
The purpose of this article is to determine the environmental impacts generated by the shops located in the settlement Ciudad Porfía in the city of Villavicencio, Colombia. The work addressed as the main technique a descriptive quantitative method, which allowed for background observation and verification; information collection was applied a survey-like tool in 133 retail establishments whose data obtained were processed using statistical tools.

Key words: sustainability, impacts, environmental practices, mitigation
\end{abstract}

\section{Introducción}

Las actividades comerciales son parte de la economía de un país, observándose a través del consumo de bienes, productos y servicios. Las organizaciones humanas establecen una intervención espacial ocasionando efectos

\footnotetext{
${ }^{1}$ Profesor, Escuela de Administración y Negocios. Facultad de Ciencias Económicas, Universidad de los Llanos, Villavicencio, Colombia. Correo electrónico: hcastro@unillanos.edu.co

${ }^{2}$ Administradora de Empresas, Universidad de los Llanos, Villavicencio, Colombia. Correo electrónico:Jenifer.pineda@unillanos.edu.co

${ }^{3}$ Profesor Titular. Facultad del Medio Ambiente y Recursos Naturales. Universidad Distrital Francisco José de Caldas. Bogotá, Colombia. Correo electrónico: jprodriguezm@udistrital.edu.co
} 
negativos por las acciones en ellas ejercidas, siendo un generador importante el sector como parte de la dimensión económica de un territorio.

Observándose que los procesos empresariales participan en el deterioro de los recursos naturales, (Feo Istúris, 2003, pág. 894) “en el mundo globalizado existen múltiples procesos que afectan directamente al ambiente y la salud", provocando cambios en los paisajes (Aledo Tur, 2008, pág.110) y la cultura de las personas en su entorno. Así mismo, se relaciona la apertura económica (Jenkins, 2003, pág.97), ya que las organizaciones buscan adaptarse a los modelos de los países desarrollados sin tener en cuenta las características propias de cada región. A su vez, uno de los mayores contaminantes son los insumos químicos (de Ita Rubio, 2003; Nadal \& Wise, 2005; Escobari, Caro \& Malky, 2004); las emisiones de SO2 (Borregaard, 2001, pág. 56) y los aceites de motor (Reátegui, 2016, pág.81), que recaen directamente en el ecosistema afectando la calidad de este.

Por otro lado, las buenas prácticas ambientales contribuyen al desarrollo sostenible, la conservación y protección del ambiente, convirtiéndose en una herramienta para los comercios, así como lo afirma (Trujillo \& Vélez Bedoya, 2006, pág. 294) “la gestión del medio ambiente puede proporcionar mejorías en el resultado económico empresarial tanto por la vía de la eficiencia y la estructura de costos, como por la vía del posicionamiento y diferenciación". En este sentido, una de las actividades que realizan las organizaciones como buenas prácticas es el reciclaje en el desarrollo de sus procesos y operaciones, además, en la implementación de una cultura ambiental con sus colaboradores (Vargas Martínez \& Olivares Linares, 2012, pág. 101).

El trabajo muestra la necesidad de implementar medidas para la prevención, conservación y mitigación de impactos ambientales que son propiciados por las pequeñas y medianas organizaciones (PyMOs) ${ }^{4}$; presentando información de referentes teóricos relacionados con impactos ambientales y buenas prácticas, así como información sobe los comercios ubicados en el asentamiento Ciudad Porfía - Villavicencio Meta, proporcionando elementos de discusión sobre los recursos afectados, tendientes a crear estrategias entre los stakeholders; seguido se plantea la metodología utilizada, posteriormente los resultados obtenidos y para finalizar las conclusiones.

\section{Metodología}

La investigación abordo de forma cuantitativa, tipo descriptivo, en donde el diseño metodológico "consiste en el conjunto más o menos coherente y racional de técnicas y procedimientos cuyo propósito fundamental apunta a implementar procesos de recolección, clasificación y validación de datos y experiencias provenientes de la realidad" (Zapata, 2013, pág. 1). Para la recolección de la información se aplicó una encuesta en 133 establecimientos de comercio; en primera instancia se efectuó un sondeo para obtener el universo, el cual fue de 203 soportado con la base de datos de la cámara de comercio de Villavicencio, luego se realizó el cálculo de la muestra probabilística para una población finita, cuyos datos obtenidos se procesaron mediante herramientas estadísticas como, el KR20, para validar el nivel de confianza del instrumento, correlación bivariada de Pearson, que permitió identificar la homogeneidad entre las variables, la matriz de Leopold, que evalúa y determina los impactos ambientales, por último una escala de contribución por sectores económicos de los impactos ambientales generados por los establecimientos de comercio.

Para el cálculo de la muestra se utilizó la siguiente fórmula:

${ }^{4}$ Término utilizado de acuerdo a la Norma ISO (International Standarization for Organization) 26000 sexta edición. 
$\mathrm{n}=\frac{Z^{2} \alpha / 2 P Q N}{\varepsilon^{2}(N-1)+Z^{2} P Q}$

Tomado de (Narváez, 2014).

Considerando un nivel de confianza del $95 \%$ y un margen de error del $5 \%$.

\subsection{Los impactos ambientales}

Se considera como la afectación del hombre al ambiente, (Espinoza, 2002, pág. 17) "el impacto es una alteración significativa de parte o la totalidad del ambiente", la cual es "producida por una actividad humana" (Garmendia, et al, 2005, pág. 17) y pueden "ser de carácter positivo o negativo" (Espinoza, 2002, pág. 25). A continuación, se relaciona una conducta de impactos ambientales como resultado de las actividades, procesos y desechos de las empresas, figura 1 Impactos ambientales como resultado de los establecimientos de comercio.

Figura 1

Impactos ambientales como resultado de los establecimientos de comercio

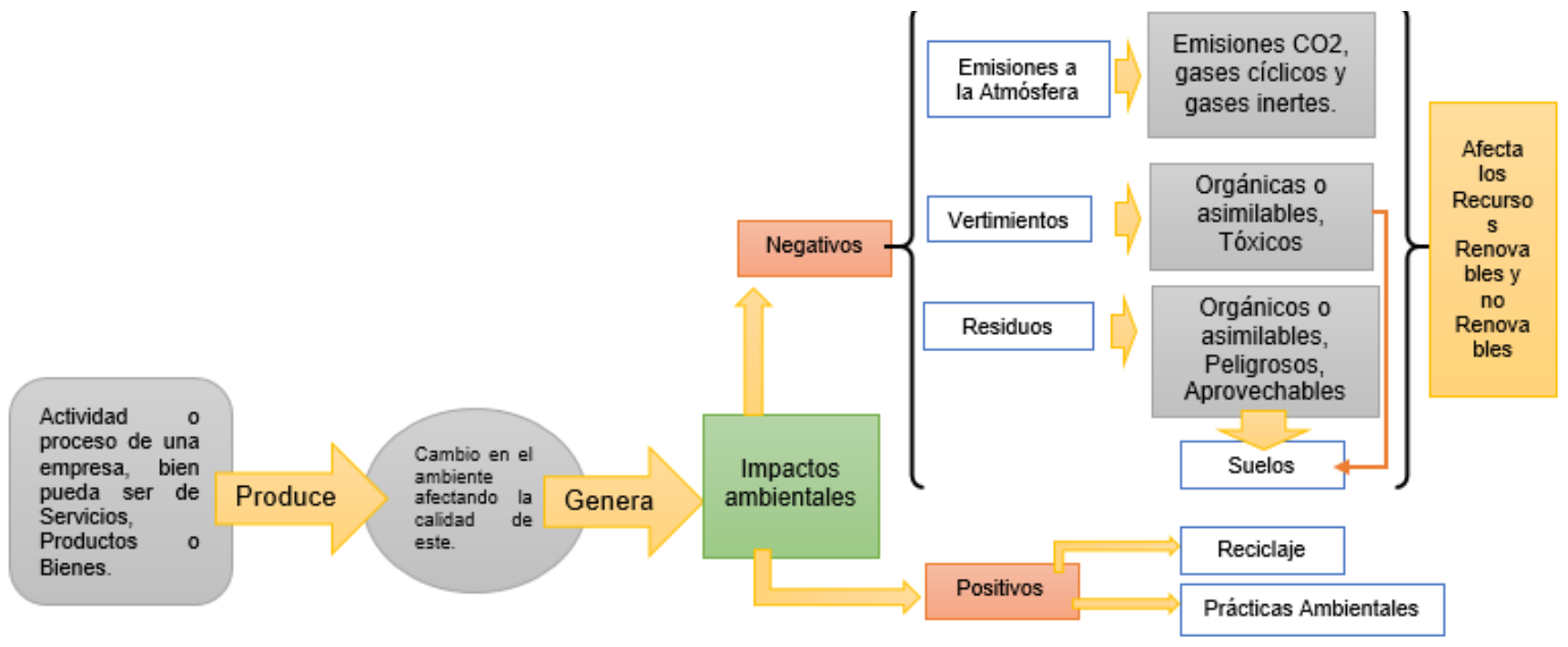

Fuente: Elaboración propia, adaptado de (Espinoza, 2002 pág. 54).

\subsection{Emisiones}

Las afectaciones de este tipo de contaminación generan efecto invernadero y deterioro de la capa de ozono al estar retenidos por la atmosfera (National Geographic, 2010, pág. 3). Produciendo sustancias que intervienen de forma negativa en el ambiente y la salud de las personas, por lo que "son conocidas como contaminantes atmosféricos" (Ministerio de Medio Ambiente, 2017, pág. 15) y de acuerdo al Instituto de Hidrología, Meteorología y Estudios Ambientales (IDEAM) (2018), es generada por la emisión, acumulación y mezcla de contaminantes en el aire provenientes de fuentes naturales (volcanes y plantas) y fuentes antropogénicas (industrias, servicios y vehículos).

\subsection{Vertimientos}

Se denominan a "la descarga final a un cuerpo de agua, a un alcantarillado o al suelo, de elementos, sustancias o compuestos contenidos en un medio líquido" (Ministerio de Medio Ambiente y Desarrollo Sostenible, 2018), es frecuente que por la inadecuada disposición "genera contaminación en las fuentes hídricas" (Segura, 2007, pág. 89); para evitar estos impactos, las CARs (Corporaciones Autónomas Regionales de Colombia) otorgan 
permisos de vertimientos "garantizando que el usuario está entregando sus aguas residuales en unas condiciones que sean aceptadas por el recurso natural" (pág.3), dado que "la disponibilidad del agua es esencial para el funcionamiento adecuado de toda organización" (Bernal Pedraza., 2010, pág. 102).

\subsection{Residuos sólidos}

De acuerdo con la GTC 53-7 de 2006 "Los residuos sólidos o desecho es cualquier objeto, material, sustancia o elemento solido resultante del consumo o uso de un bien en actividades domésticas, industriales, comerciales, institucionales, de servicios, que el generador abandona, rechaza, o entrega". Es así que se clasifican en aprovechables y no aprovechables:

Figura 2

Residuos sólidos

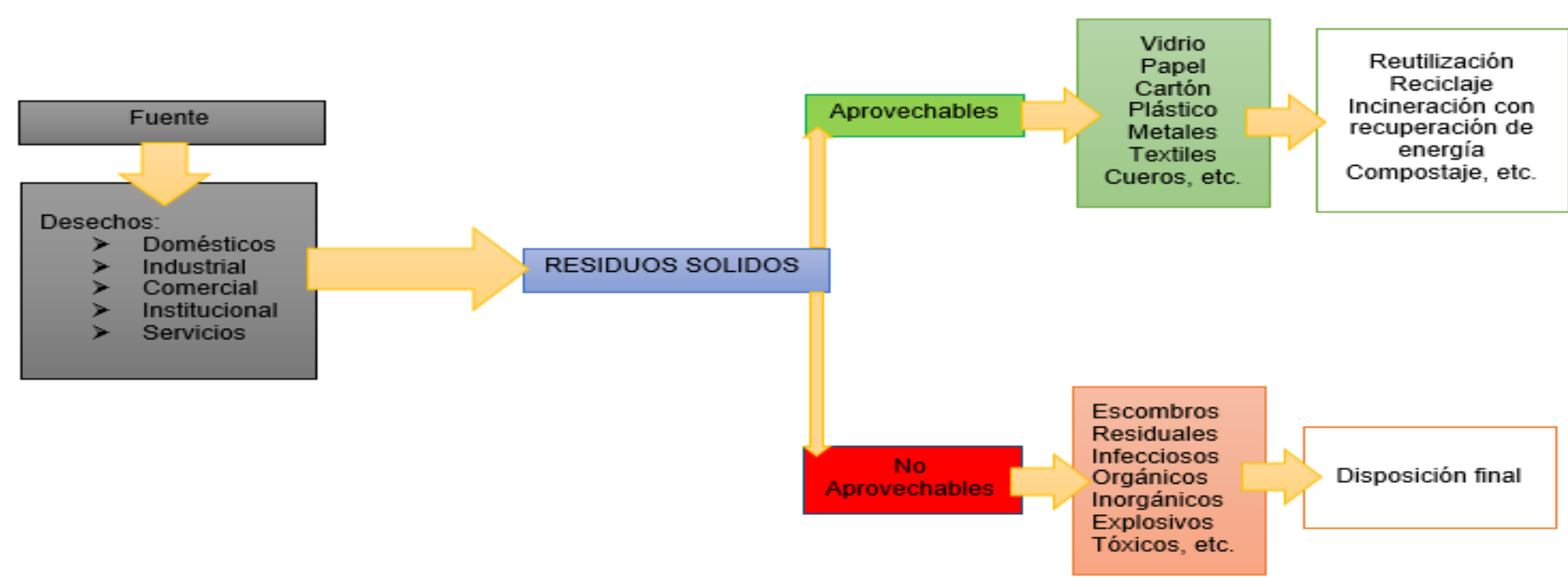

Fuente: Elaboración propia, adaptado de GTC 53-7 (2006)

La condición de manejo de los residuos sólidos en Colombia es precaria, "la disposición final se realiza con poco control en la mayoría de los municipios, ocasionando contaminación ambiental” (Puerta, 2004, pág. 57). Y según datos del IDEAM para el año 2017 en Colombia se produjo 489.057,3 toneladas de residuos peligrosos, de acuerdo a las actividades productivas CIIU (Clasificación Industrial Internacional Uniforme).

\subsection{Prácticas ambientales}

Son aquellas actuaciones amigables con el entorno, (Linea verde Torrelavega, s.f.) "son acciones que pretenden reducir el impacto ambiental negativo que causan los procesos" (pág.1) y según (Conde, 2015, pág. 1) van encaminadas a: optimizar el consumo de recursos naturales, disminuir la producción de sustancias contaminantes, minimizar y gestionar adecuadamente los residuos que se producen durante la actividad, sensibilizar y educar ambientalmente tanto a los trabajadores.

\subsection{Reciclaje}

Una de las apuestas para minimizar los impactos ambientales y la reutilización de productos de un solo uso es el reciclaje, "involucra los procesos mediante los cuales se aprovechan y transforman los residuos sólidos recuperados y se devuelven a los materiales sus potencialidades de reincorporación como materia prima para la fabricación de nuevos productos" (Gómez, 2000, pág. 44). Así mismo, cuenta con tres etapas las cuales son: "recuperación, transformación y comercialización de los residuos reutilizables" (Lenis Ballesteros, López Arango \& Cuadros Urrego, 2012, pág. 868); convirtiéndose en una apuesta para mitigar el impacto que se puede causar por una inadecuada disposición y/o utilización desmesurada de los recursos naturales. 


\subsection{Microempresas en el Municipio de Villavicencio y el Barrio Ciudad Porfía.}

En Colombia la participación empresarial se clasifica en micro, pequeña, mediana y gran empresa, reglamentada por la Ley 590 de 2000 y sus modificaciones (Ley 905 de 2004 y Ley 1450 de 2011). En el artículo 43 de la Ley 1450 de 2011 define a empresa como toda unidad de explotación económica, realizada por persona natural o jurídica, en actividades agropecuarias, industriales y comerciales o de servicios, en el área rural o urbana. La segmentación está "catalogada de acuerdo al número de trabajadores, valor de las ventas brutas anuales y valor de activos totales" (Bancoldex, 2019); para el caso de las micro empresas están bajo los parámetros de número de trabajadores no superior a 10 y activos totales inferiores a 500 Salarios mínimos legal mensual vigente (SMLMV), ver tabla 1.

Tabla 1

Clasificación de las empresas en Colombia

\begin{tabular}{lcc}
\hline Segmentación empresarial & Número de trabajadores & Activos Totales \\
Micro & No superior a 10 & Inferior a 500 SMLMV \\
Pequeña & Entre 11 y 50 & Entre 501 y 5.000 SMLMV \\
Mediana & Entre 51 y 200 & Entre 5.001 y 30.000 SMLMV \\
Gran Empresa & Superior a 200 & Superior a 30.000 SMLMV
\end{tabular}

Fuente: Elaboración propia a partir de Ley 905 de 2004

Sin embargo, el Gobierno Nacional expidió el Decreto 957 de 2019 adicionando al Decreto 1074 de 2015 del Ministerio de Comercio, Industria y Turismo (MINCIT); en el cual, "se establece una nueva clasificación de tamaño empresarial basada en el criterio único de ingresos por actividades ordinarias" (MINCIT, 2019) y empieza a regir en diciembre del mismo año. Ver tabla 2.

Tabla 2

Clasificación de las empresas en Colombia por Sectores

\begin{tabular}{lccc}
\hline Tamaño Empresarial & Manufactura & Servicios & Comercio \\
Micro & Hasta 811 & Hasta 1.131 & Hasta 1.535 \\
Pequeña & Desde 811 hasta 7.027 & Desde 1.131 hasta 4.523 & $\begin{array}{c}\text { Desde } 1.535 \text { hasta } \\
14.781\end{array}$ \\
Mediana & Desde 7.027 hasta & Desde 4.523 hasta & Desde 14.781 hasta \\
& 59.527 & 16.558 & 74.066 \\
Gran Empresa & Desde 59.527 & Desde 16.558 & Desde 74.066 \\
\hline ** Cifras de ingresos por actividades ordinarias anuales en millones de pesos colombianos de 2019. \\
\end{tabular}

El municipio de Villavicencio a corte de 25 de julio de 2019 cuenta con una totalidad de 24.710 unidades empresariales de acuerdo a información suministrada por Cámara de comercio de Villavicencio; a su vez, las actividades con mayor presencia son el comercio al por mayor y menor, servicios, alojamiento, productos alimenticios, artículos de servicios personales, construcción, transporte y entre otros; asimismo las microempresas tienen una participación del $94,42 \%$ como se evidencia en la tabla 3. 
Tabla 3

Distribución económica en Villavicencio por tamaño.

\begin{tabular}{lcc}
\hline Tamaño Empresarial & $\begin{array}{c}\text { Número de } \\
\text { establecimientos }\end{array}$ & $\begin{array}{c}\text { Porcentaje de } \\
\text { participación }\end{array}$ \\
Micro & 23.330 & $94,42 \%$ \\
Pequeña & 1.070 & $4,33 \%$ \\
Mediana & 257 & $1,04 \%$ \\
Gran Empresa & 53 & $0,21 \%$ \\
\hline
\end{tabular}

Fuente: Elaboración propia a partir de Cámara de Comercio de Villavicencio 2019.

En cuanto al Barrio Ciudad Porfía se evidencia una participación del 3,04\% con 751 unidades empresariales, del total de establecimientos comerciales encontrados en este sector el 98,93\% corresponde a microempresas, cuyas actividades están relacionadas con el comercio al por mayor y menor, alojamiento, productos alimenticios, entre otros. En la tabla 4 se observa la distribución por tamaño empresarial en el área de estudio.

Tabla 4

Distribución económica en el Barrio Ciudad Porfía

\begin{tabular}{lcc}
\hline Tamaño Empresarial & $\begin{array}{c}\text { Número de } \\
\text { establecimientos }\end{array}$ & $\begin{array}{c}\text { Porcentaje de } \\
\text { participación }\end{array}$ \\
Micro & 743 & $98,93 \%$ \\
Pequeña & 6 & $0,80 \%$ \\
Mediana & 2 & $0,27 \%$ \\
Gran Empresa & 0 & $0 \%$ \\
\hline
\end{tabular}

Fuente: Elaboración propia a partir de Cámara de Comercio de Villavicencio 2019.

\section{Resultados}

El estudio se concentró en las microempresas dado su porcentaje de participación en el centro poblado analizado.

Se realizó el análisis del nivel de confiablidad del instrumento obteniéndose 0,75 en el KR20 (ver tabla 5), esto significa que cuenta con un aceptable nivel de confianza, por tanto, el instrumento es viable para ser aplicado.

Tabla 5

Estadística de fiabilidad (KR20)

KR20 N de elementos

$0,750 \quad 20$

Fuente: Elaboración propia

Al examinar la correlación bivariada de Pearson en el Programa SPSS (Statistical Product and Service Solutions) que permitió identificar la homogeneidad entre los criterios definidos en la investigación (Rangos de interpretación de la correlación: 1 Perfecta; 0,81-0,99 Alta; 0,61-0,80 Medio Alta; 0,41-0,60 Media; 0,21 0,40 Medio Baja; 0,01 - 0,20 Baja; 0 Nula; ver tabla 8) y la evaluación de los impactos ambientales a través de la Matriz de Leopold (ver Anexo 1) se evidenció en la tabla 6. 
Tabla 6

Estadístico de correlación (Correlación de Pearson)

Correlaciones

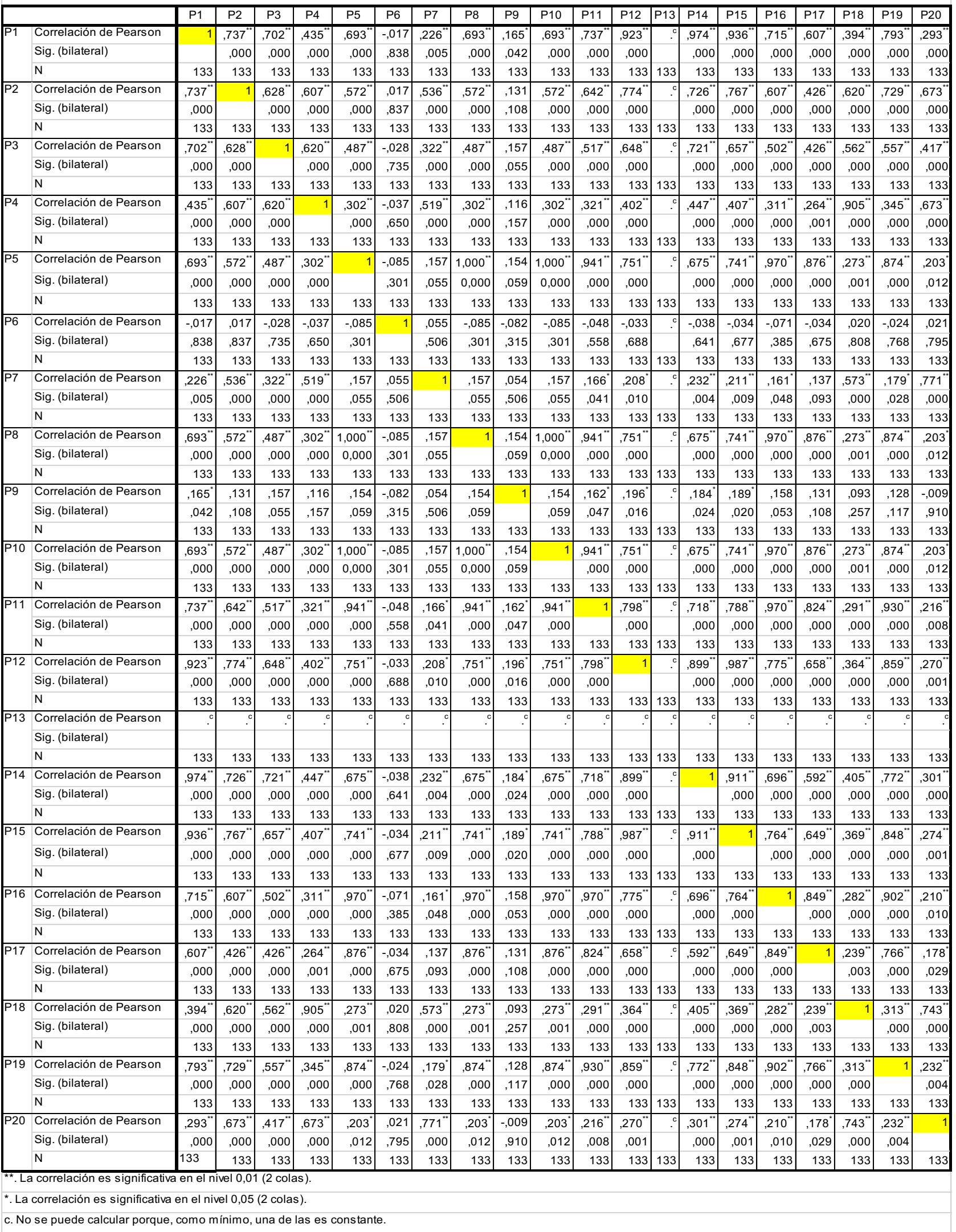

Fuente: Elaboración propia a partir del SPSS8. 
El 3,16\% de los comercios encuestados generan emisiones, por tanto, su impacto al ambiente es negativo, son causados por la producción, comercialización y venta de sustancias agotadoras de la capa de ozono y químicos en los establecimientos de comercio, tales como aerosoles, productos de aseo (clorox, limpiadores de pisos), productos cosméticos (plomo, mercurio), entre otros; ya que contienen material particulado como el dióxido de azufre (SO2) y el dióxido de nitrógeno (NO2).

El 3,56\% de los comercios encuestados presenta vertimientos, con impacto ambiental negativo, especialmente en el manejo de aguas residuales por parte de los establecimientos de comercio, dado que su disposición final es el rio Ocoa causando contaminación y pérdida de las condiciones naturales en esta fuente hídrica y su entorno. Es importante resaltar, que las acciones realizadas por estos actores están asociados a patrones culturales. Resaltando que, el $78,77 \%$ de la muestra implementa medidas de eficiencia en los recursos para reducir el uso de energía, agua y otros recursos.

El 23,32\% de los comercios encuestados contribuyen con un adecuado manejo de residuos sólidos, por tanto, presenta un impacto negativo bajo, debido a una correcta disposición, pero, es importante contrastar que el $62,33 \%$ no realiza clasificación en la fuente, no reutiliza ni hace tratamiento antes de su disposición final. Por otra parte, los que producen los establecimientos de comercio en la avenida principal del barrio Ciudad Porfía (tabla7).

Tabla 7

Residuos sólidos generados por los establecimientos de comercio de la avenida principal del Barrio Ciudad Porfía.

\begin{tabular}{lll}
\hline Ítem & Residuo Sólido & Porcentaje \\
\hline 1 & Biodegradables & $28 \%$ \\
2 & Reciclables & $17 \%$ \\
3 & Inertes & $13 \%$ \\
4 & Ordinarios & $35 \%$ \\
5 & Peligrosos & $7 \%$ \\
& Total & $\mathbf{1 0 0 \%}$ \\
\hline
\end{tabular}

Fuente: Elaboración propia

En cuanto a las prácticas ambientales se relaciona un impacto al ambiente negativo medio con un $43,48 \%$, es decir, los establecimientos de comercio no implementan medidas orientadas a la prevención, reducción y/o eliminación de contaminantes que afectan directa e indirectamente su entorno, el 26,48\% de ellos no separan los desechos que producen sus actividades (figura 3).

Figura 3

Impactos ambientales generados por actividades de los establecimientos de comercio en la Avenida principal del Barrio Ciudad Porfía en puntos de acuerdo a la Matriz de Leopold.

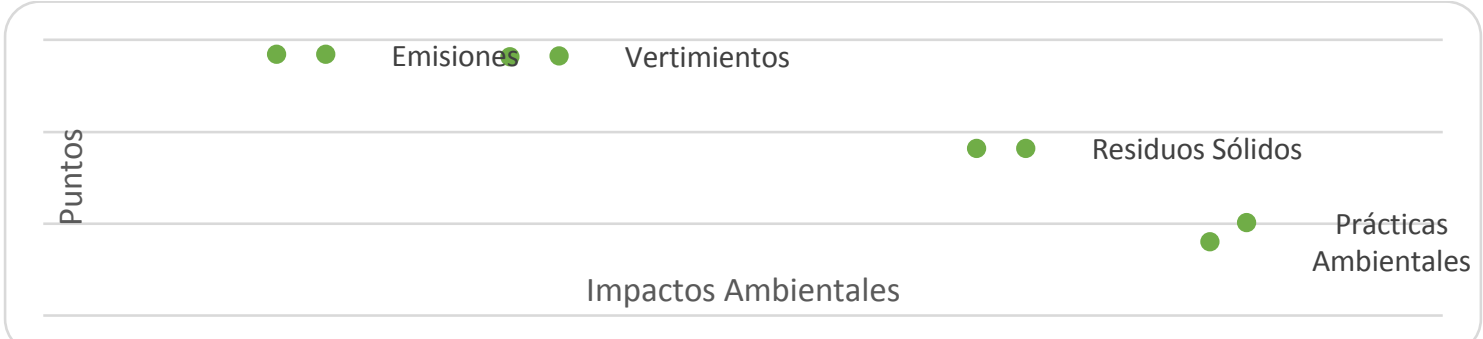

Fuente: Elaboración propia 
La composición de actividades económicas del sector de estudio cuenta con una participación de establecimientos del sector terciario, es decir, actividades enfocadas a la prestación de servicios, ver figura 4; y se determina que las actividades que generan mayor impacto son las relacionadas con el comercio al por menor (supermercados, misceláneas, ferreterías, prendas de vestir, calzado, productos de farmacia, compraventas, venta de celulares y ópticas) y actividades de servicios de comidas y bebidas (restaurantes, panaderías y cafeterías), ver tabla 8.

Figura 4

Representación de los establecimientos de comercio por actividades económicas en la Avenida principal del barrio Ciudad Porfía

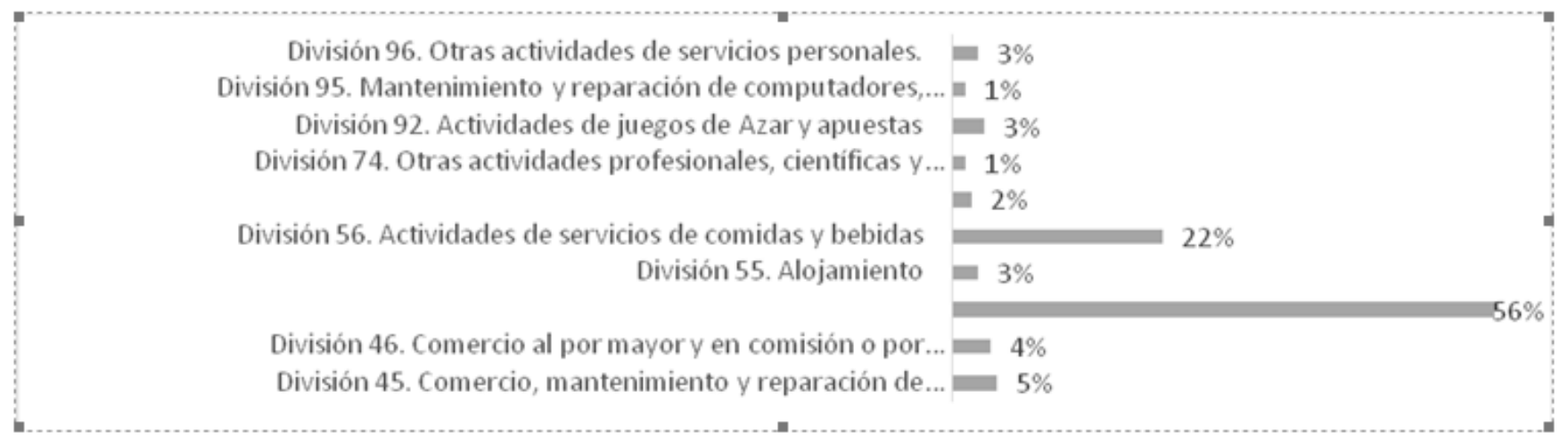

Fuente: Elaboración propia a partir de datos del CIIU.

\subsection{Discusión}

Para (Arregui, 2006; Cabrera \& González, 2005; y Pichardo, 2009) afirman que el deterioro ambiental se debe a la producción y uso desmesurado de componentes nocivos, contaminación, vertimientos y destrucción de bosques por parte de las empresas y que no existen políticas claras del gobierno en la prohibición y control de estos para mitigar el impacto. Asimismo, Prieto \& Abraham (2000, pág. 4) indican que el uso continuado produce efectos y cambios irreversibles en el entorno. Además, es importante resaltar que las empresas hoy día para que tengan una "ventaja competitiva" (Trujillo \& Vélez, 2006) deben implementar medidas en sus procesos para la mitigación de impactos.

De acuerdo con los resultados del estudio, los impactos que generan los comercios son: Residuos Sólidos, Emisiones y vertimientos; esto porque se encuentra relacionado con un manejo subjetivo de las actividades económicas, así como la falta de presencia institucional.

Ramírez Hernández (2005, pág. 8) pone de manifiesto que existe una relación sociedad-naturaleza, en donde "se reconoce un complejo sistema de relaciones intrínsecamente conectadas, donde el bienestar y la protección de unas condiciones favorables para la especie, conllevan necesaria y vitalmente a la protección, preservación y restablecimiento del entorno natural"; por ende, (Vargas \& Olivares, 2012; Moreno, 2005) aducen que para una mayor preservación ambiental por parte de las organizaciones, debe incluirse a los empleados y comunidad para generar una cultura en pro del beneficio del ecosistema y así contribuir de forma positiva con buenas prácticas ambientales. Se determinó que dichos impactos mencionados, se originan por patrones culturales, es decir, no implementan medidas eficientes para la mitigación, control y preservación; a su vez, no dimensionan los efectos nocivos que provocan en los ecosistemas aledaños por la producción, comercialización y/o uso de los bienes o servicios que le ofrecen a la comunidad. 


\section{Conclusiones}

Se determinó que las microempresas cumplen una función importante en el desarrollo y crecimiento económico de cualquier comunidad, son generadores de empleo y crecimiento urbanístico, además, influye en el mejoramiento de la calidad de vida de las personas y la valorización de los predios. Los comercios ubicados en la avenida principal del barrio Ciudad Porfía son generadores de impactos ambientales, en su mayoría se clasifican de orden negativo.

Las actividades con mayor participación en la generación de impactos en este sector son: comercio al por menor con un $59,76 \%$ y Servicios de comida y bebidas con un 16,72\%. Comprendiendo la comercialización y uso inadecuado de sustancias químicas, así como la disposición final de residuos sólidos de manera inadecuada en fuentes hídricas, tanto como en el espacio público.

Se estableció que debido a los patrones culturales se refleja una conducta repetitiva frente al manejo deficiente de los bienes y servicios comercializados; adicionalmente se adolece de conocimiento acerca de la generación de impactos negativos al entorno y su manejo; así como la ausencia de control en el cumplimiento de la normativa ambiental es determinante en la gestión adecuada tanto de residuos sólidos como de vertimientos.

\section{Referencias bibliográficas}

Aledo Tur, A. (2008) De la tierra al suelo: La transformación del paisaje y el nuevo turismo residencial. En: Arbor: Ciencia, Pensamiento y Cultura. Vol. 184, no 729, p. 99-113 (110).

Arregui, O. (2006). Sostenibilidad y estudios de impacto. En: Revista Virtual Universidad Católica del Norte. Vol. 12 , no 3 , p. $1-12$

Artículo 43 Ley 1450 Congreso De La República de Colombia. (2011). Definiciones de tamaño empresarial. Colciencias. Bogotá D.C., 2018. 89 p.

BANCO DE COMERCIO EXTERIOR DE COLOMBIA S.A. (BANCOLDEX). Clasificación de empresas en Colombia. [Sitio web]. Bogotá D.C. [consultado: 02 de agosto de 2019]. Disponible en: https://www.bancoldex.com/sobre-bancoldex/quienes-somos/clasificacion-de-empresas-en-colombia23444.

Bernal Pedraza, AY. (2010). Gestión del agua - una preocupación de las empresas ambientalmente responsables. En: Universidad \& Empresa. Julio-diciembre, 2010. Vol. 12, no 19, p. 87-106.

Borregaard, N. (2001). Valorización económica de los impactos ambientales en la minería chilena. Ambiente y Desarrollo, 17(1), 50-58.

Cabrera, E. D. M., \& González, C. B. (2005). Impacto ambiental de sustancias agotadoras de ozono. Tecnología Química, 25(2), 21-25.

CAR's- Corporaciones Autónomas Regionales de Colombia. (2012). Permiso de vertimientos. Recuperado de https://www.car.gov.co/vercontenido/1168

Conde, JM. (2015). Buenas prácticas ambientales [en línea]. Conde formación. Isla Santa Cruz, España. [Consultado: 25 de junio de 2019]. Disponible en: http://www.condeformacion.com/manual-de-buenaspracticas-ambientales/.

de Ita Rubio, A. (2003). Los impactos socioeconómicos y ambientales de la liberalización comercial de los granos básicos en el contexto del TLCAN: El caso de Sinaloa. Centro de estudios para el cambio en el campo mexicano, Centro Mexicano de Derecho Ambiental. 53 p. 
Escobari, J., Caro, V., \& Malky, A. (2004). Problemática ambiental en Bolivia. Documento de trabajo. La Paz, Bolivia. Unidades de análisis de políticas sociales y económicas. $31 \mathrm{p}$.

Espinoza, G. A. (2002). Gestión y fundamentos de evaluación de impacto ambiental. BID/CED. técnica No ATN/JF-6618-RG "Programa de apoyo para el mejoramiento de la gestión ambiental en los países de América Latina y el Caribe". Santiago de Chile.

Feo Istúriz, O. (2003). Reflexiones sobre la globalización y su impacto sobre la salud de los trabajadores y el ambiente. Ciência \& Saúde Coletiva, 8(4), 887-896.

Garmedia, A., Salvador, A., Crespo, C., \& Garmendia, L. (2005). Evaluación de impacto ambiental. Madrid: Pearson Educación, S.A., 2005. 416 p. ISBN: 84-205-4398-5.

Gómez, C. I. S. (2000). Problemática y gestión de residuos sólidos peligrosos en Colombia. INNOVAR. Revista de Ciencias Administrativas y Sociales, (15), 41-52. Doi: ISSN electrónico 2248-6968.

GTC - Guía Técnica Colombiana. (2006). Guía para el aprovechamiento de residuos sólidos orgánicos no peligroso. Guía Técnica COLOMBIANA 53-7 2006-04-26. Instituto Colombiano De Normas Técnicas Y Certificación. Bogotá D.C.: El Instituto. 2006. 24 p.

Hernández, O. J. R. (2005). Responsabilidad, ética y evaluación de impacto ambiental. Revista Luna Azul, (21). Vol. 12, no 1, p. 1-9.

IDEAM- Instituto de Hidrología, Meteorología y Estudios Ambientales. (2018). Emisiones, recuperado de http://www.siac.gov.co/emisionesaire\#: :text=Emisiones\%20aire\%20\%2D\%20IDEAM\&text=La\%20contam inaci\%C3\%B3n\%20atmosf\%C3\%A9rica\%20es\%20generada,industrias\%2C\%20servicios\%20y\%20veh\%C3\%A Dculos).

Jenkins, R. O. (2003). La apertura comercial¿ ha creado paraísos de contaminadores en América Latina?. Revista de la CEPAL.. Vol. 1, no 80, p. 85-100 (97).

Ley 590 Congreso De La República de Colombia. (2000). Por la cual se dictan disposiciones para promover el desarrollo de las micro, pequeñas y medianas empresa. Colciencias. Bogotá D.C., 2018. 44 p.

Ley 905 Congreso De La República de Colombia. (2004). Por medio de la cual se modifica la Ley 590 de 2000 sobre promoción del desarrollo de la micro, pequeña y mediana empresa colombiana y se dictan otras disposiciones. Colciencias. Bogotá D.C., 2018. 22 p.

Lenis Ballesteros, V., López Arango, Y. L., \& Cuadros Urrego, Y. M. (2012). Condiciones de salud y de trabajo informal en recuperadores ambientales del área rural de Medellín, Colombia, 2008. Revista de Saúde Pública, 46(5), 866-874. Doi: http://dx.doi.org/10.1590/\$0034-89102012000500014.

Linea Verde Torrelavega. Guías de buenas prácticas sobre medio ambiente [sitio web]. Cantabria, España. [Consultado: 20 junio de 2019]. Disponible en: http://www.lineaverdetorrelavega.com/lv/guias-buenaspracticas-ambientales/introduccion-buenas-practicas-ambientales/que-es-el-consumo-responsable.asp.

Ministerio De Ambiente Y Desarrollo Sostenible. (2015). Presenta nueva Norma de Vertimientos que permitirá mejorar la calidad agua del país [sitio web]. Minambiente. Bogotá, D.C., Consultado: 20 de junio de 2019]. Disponible en: http://www.minambiente.gov.co/index.php/noticias/1700-minambiente-presenta-nuevanorma-de-vertimientos-que-permitira-mejorar-la-calidad-agua-del-pais.

Ministerio De Ambiente Y Desarrollo Sostenible. (2017). Guía para la elaboración de inventarios de emisiones atmosféricas. Coordinado por Mauricio Gaitán Varón y Paula Andrea Cárdenas Ruiz. Bogotá, D.C.: Dirección de Asuntos Ambientales, Sectorial y Urbana, 2017. 92 p. ISBN: 978-958-8901-64-0 
MINCIT -Ministerio De Comercio, Industria Y Turismo (2019). Gobierno expide nueva clasificación de empresas a partir de sus ingresos [sitio web]. Bogotá D.C. Disponible en:

http://www.mincit.gov.co/prensa/noticias/industria/gobierno-expide-nueva-clasificacion-de-empresas-a.

MINCIT -Ministerio De Comercio, Industria Y Turismo (2019). Decreto 957 Por el cual se adiciona el capítulo 13 al Título 1 de la Parte 2 del Libro 2 del Decreto 1074 de 2015, Decreto Único del Sector Comercio, Industria y Turismo y se reglamenta el artículo $2^{\circ}$ de la Ley 590 de 2000, modificado por el artículo 43 de la Ley 1450 de 2011. Noticia de Industria. Bogotá D.C., 2019. 7 p

Moreno, F. F. L. (2005). La empresa ambientalmente responsable. Una visión de futuro. Economía, (21), 3958.Vol. 20, no 1, p. 39-58.

Nadal, A. T., \& Wise, A. (2005). Los costos ambientales de la liberalización agrícola: El comercio de maíz entre México y EE. UU. en el marco del NAFTA. En globalización y medio ambiente: lecciones desde las Américas. Publicado por Heinrich Böll Foundation North América, 49-92.

Narváez, GA. (2014). Muestra en la investigación cuantitativa [en línea]. Slideshare.net. Pasto. [Consultado: 20 de febrero de 2019]. Disponible en: https://es.slideshare.net/gambitguille/muestra-en-la-investigacincuantitativa.

National Geographic. (2019). Disminución del ozono [sitio web]. EEUU. (5, septiembre, 2010). Disponible en: https://www.nationalgeographic.es/medio-ambiente/disminucion-del-ozono.

Pichardo Pagaza, I. (2009). Responsabilidades municipales en materia ambiental. Convergencia, 16(49), 291308.

Prieto, M. D. R., \& Abraham, E. M. (2000). Caminos y comercio como factores de cambio ambiental en las planicies áridas de Mendoza (Argentina) entre los siglos XVII y XIX. Theomai, (2).

Puerta Echeverreria, SM. (2004). Los residuos sólidos municipales como acondicionadores de suelo. En: Revista Lasallista de Investigación. Enero-junio, Vol. 1, no 1, p. 56-65.

Reátegui, K. (2016). Lavado de vehículos automotores en la ciudad y su efecto sobre el uso del agua y el ambiente Loreto-2016. Tesis Ingeniero en Gestión Ambiental, Iquitos, Perú. Universidad Nacional de la Amazonia Peruana. Facultad de Agronomía, Escuela Profesional de Ingeniería en Gestión Ambiental, 81 p.

Segura, LE. (2007). Estudio de antecedentes sobre la contaminación hídrica en Colombia. Trabajo de grado Administrador Público, Bogotá, D.C. Escuela Superior de Administración Pública (ESAP). Facultad de Pregrado, Programa en Ciencias Políticas y Administrativas. 99 p.

Trujillo, M. A., \& Vélez Bedoya, R. (2010). Responsabilidad ambiental como estrategia para la perdurabilidad empresarial. Revista Universidad y Empresa, 8(10), 291-308.. Doi: ISSN: 0124-4639

Vargas Martínez, E. E., \& Olivares Linares, A. A. (2012) Responsabilidad empresarial: el caso de" Hotelera Posadas".. Responsabilidad ambiental empresarial: el caso de "Hotelera Posadas". En: El Periplo Sustentable. Vol. 1, no 22, p. 63-188. Doi: ISSN: 1870-9036

Zapata, S. (2013) ¿Cómo redactar la Metodología o Diseño Metodológico? [en línea]. Seminario Monográfo. Nicaragua. Disponible en: https://seminariomonografico.blogspot.com/2013/05/como-redactar-lametodologia-o-diseno.html

Esta obra está bajo una Licencia Creative Commons Attribución-NoCommercial 4.0 International

(c) EY-NG 